\title{
On call paediatricians were not better than advice nurses for after hours medical advice in a general paediatric population
}

Lee TJ, Baraff U, Guzy J, et al. Does telephone triage delay significant medical treatment? Advice nurse service vs on-call pediatricians. Arch Pediatr Adolesc Med 2003;157:635-41.

Q In a general paediatrics population, is medical advice from on call paediatricians compared with that from advice nurses associated with (1) fewer parents or guardians seeking unadvised medical care, and (2) fewer parents or guardians obtaining unadvised significant care for their children?

\section{METHODS}

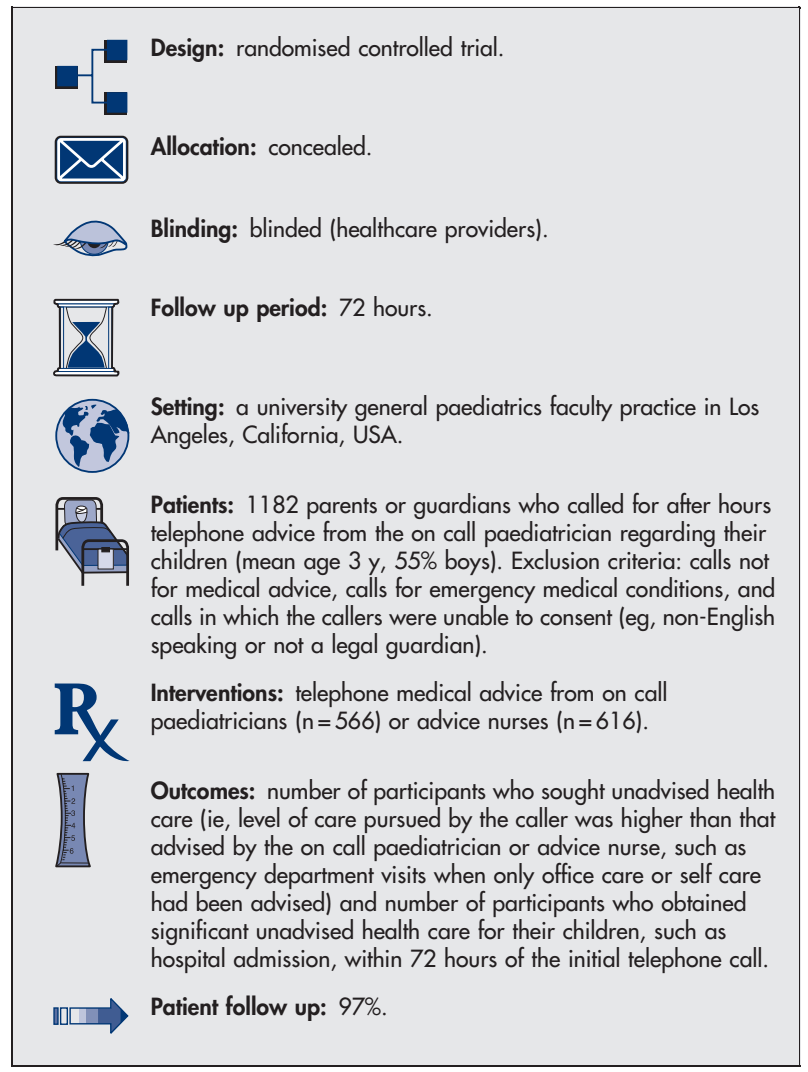

\section{MAIN RESULTS}

The groups did not differ for number of participants who sought unadvised health care or number of participants who sought and obtained significant unadvised health care for their children (table).

For correspondence: Dr L J Baraff, Emergency Medicine Center, UCLA Medical School, Los Angeles, CA, USA. Ibaraff@ucla.edu

Source of funding: Agency for Healthcare Research and Quality.

\section{CONCLUSION}

In a general paediatrics population, medical advice from on call paediatricians or advice nurses did not differ for number of parents or guardians who sought unadvised medical care or those who obtained unadvised significant medical care for their children.

\section{Commentary}

The tension between cost effective and safe care is played out nightly on the telephone when anxious parents call asking what to do about

a child's presumed illness. The network of after hour, telephone advice services using protocols to direct decision making has grown exponentially in North America in the past decade. 'In the UK, National Health Service Direct was launched in 1998, and $40 \%$ of calls concern children. ${ }^{2}$ Previous studies have found appropriate rates of referral to emergency departments and low risk of morbidity and mortality after telephone advice provided by non-physicians who used clinical algorithms. ${ }^{3}$ However, the study by Lee et al contributes to our understanding of this phenomenon by examining an important subset of the advice seeking population: those who are deemed "well enough" to delay care. This study attempted to ascertain the safety of advice given by nurses to delay care or to provide self care compared with that given by paediatricians. The results suggest that nurses using triage algorithms are safe substitutes for physicians. In the 2 cases where nursing advice was followed up by significant unadvised care that included hospital admission, detailed clinical summaries suggest that hospital admission was not indicative of poor advice from nurses.

It is important to recognise the context of the study. The sample was drawn from a highly educated, privately insured population accustomed to having a paediatrician available for their after hours calls. Further studies should be done in populations that are less educated and have fewer resources. Studies with parents who are less knowledgeable and have poorer communication skills may reveal differences not apparent in this study.

Elizabeth Hawkins-Walsh, RN, DNSc, CPNP, MCHN The Catholic University of America Washington, DC, USA

1 Anders G. Telephone triage: how nurses take calls and control the care of patients from afar. Wall Street Journal February 4, 1997;A1.

2 Monaghan R, Clifford C, MCDonald P. Seeking advice from NHS direct on common childhood complaints: does it matter who answers the phone? J Adv Nurs 2003;42:209-16.

3 Barber JW, King WD, Monroe KW, et al. Evaluation of emergency department referrals by telephone triage. Pediatrics 2000;105:819-21.

On call paediatricians $v$ advice nurses for after hours medical advice in a general paediatric population*

\begin{tabular}{lllll}
\hline Outcomes at $\mathbf{7 2}$ hours after the initial call & On call paediatrician & Advice nurses & RRI (95\% Cl) & NNH \\
\hline Sought unadvised care & $20 \%$ & $19 \%$ & $4.5 \%(-18$ to 33$)$ & Not significant \\
\hline & & & RRR (CI) & NNT \\
\hline $\begin{array}{l}\text { Sought and obtained unadvised significant } \\
\text { care }\end{array}$ & $4.2 \%$ & $4.3 \%$ & $2.1 \%(-69$ to 43$)$ & Not significant \\
\hline
\end{tabular}

*Abbreviations defined in glossary; RRI, RRR, NNH, NNT, and Cl calculated from data in article. 